\title{
Un nouveau mandat, des lecteurs plus nombreux, des exigences accrues
}

Commele rappellejudicieusement Bernard Charlin, premier rédacteur en chef et co-fondateur de la revue, le lancement de "Pédagogie médicale » a d'abord résulté de l'initiative volontariste de trois passi onnés. Engagés dans leurs institutions respectives et préoccupes de corriger cette anomalie que constituait I'absence de tribune francophone au service d'une communauté qui, depuis plusieurs années déà, fourmillait pourtant d'initiatives fécondes, d'expériences remarquables et d'expertises reconnues, ilsn'ont ménagé ni leur temps, ni leurs énergies à la réussite du projet. Des fées bienveillantes se sont d'emblée penchées sur son berceau. La Conférence Internationale des $D$ oyens des Facultés de $M$ édecine d'Expression Française (CIDM EF) a notamment été de celles là et n'a pas épargné son soutien moral et financier, constamment renouvelé depuis. D'autres organi smes et institutions l'ont rejointe dès le début et ont, à la mesure de leurs moyens et de leurs missions respectifs, apporté des contributions originales, notamment d'ordre éditorial. Gérard $D$ ongradi et $M$ ireille $M$ énestrel, à travers leur socié té Acalis, ont pour leur part pleinement joué leur rôle d'éditeur, et non pas simplement ceux d'imprimeur et de diffuseur, en soutenant activement le projet et en assumant, avec loyauté et compétence, ses risques et ses vicissitudes. Enfin, les auteurs de travaux, en confiant leurs manuscrits à un média d'audience encore incertaine, ainsi que les abonnés, ont témoigné leur confiance envers l'entreprise et démontré du même coup la justesse du pari initial.

L'ensemble de l'édifice restait cependant fragile. II y manquait la visibilité, la légitimité et le crédit, que seule pouvait apporter une grande société savante. C'est chose faite avec la création de la Société Internationale Francophone d'Education M édicale (SIFEM), dont "Pédagogie M édicale » devient désormaisl'organe officiel d'expression. U n tel mandat apporte la promesse d'une plus grande reconnaissance et d'une audience plus vaste ; il confère aussi à l'équi pe qui en reçoit la charge des responsabilités nouvelles. Je mesure la confiance et I'honneur qui me sont signifiés par la communauté francophone d'éducation médicaleen me dél éguant la fonction de rédacteur en chef. Avec dévouement, un comité de rédaction engagé et qui ne comptera pas son temps, encouragé et éclairé par un comité éditorial récemment renouvelé sous la houlette de Raymond Colin, sattachera à rendre compte de la vitalité de la péda gogie médicale francophone.
La nécessité que les enseignants sinterrogent systé matiquement sur le niveau de "preuve»-ou en tout cas de rigueur- que possedent les savoirs sur lesquels ils fondent leursactions est un acquis de ces dernières années, dont témoigne, par exemple, le courant Best Evidence $M$ edical Education. L a revues'efforcera d'apporter une contribution croissante à cet égard, en accueillant les travaux originaux de la communauté francophone et en sollicitant, auprès de personnes-ressources reconnues, des synthèses didactiques et des revues systématiques de la littérature sci entifique concernant les grandes questions de l'éducation médicale. Pour autant, nous garderonsà l'esprit que, à l'instar de la médecine, la pédagogie n'est pas une science mais reste bien une pratique enseignante complexe, accompagnée de science et de technique. Le journal continuera ains à rendre compte des experiences concrètes développées au sein de nos différents facultés, écoles ou instituts, en formation initiale commeen formation continue, pour toutes les professions de santé, pour autant qu'elles soient rapportées avec le souci de livrer, au-delà du particularisme de chaque situation, un message pédagogique de portée genérale.

M ais, même lorsqu'elle est mise en cuvre au niveau de l'excellence, la pédagogie médicale n'est pas une fin en soi. Elle n'acquiert sa légitimité que lorsqu'elle se met au service du développement de l'expertise des professionnels, dans le but de contribuer à améliorer létat de santé des personnes et des populations concernées. D ans cettelivraison du journal, Charles Boelen nous rappelle fort opportunément les enjeux dela responsabilité sociale des enseignants en sciences de la santé et un texte collectif de grande portée resitue le professionnalisme médical dans la perspective culturelle du contrat social qui lie médecine et socié té. Ces orientations doivent être, à nos yeux, des points d'ancrage forts pour notre revue. A cet égard, nous nous efforcerons de rendre compte que les problémati ques croi sées de la recherche en éducation, de la formation des enseignants et de l'évaluation des pratiques pédagogiques, ne sont pas simplement d'ordre méthodologique (quels moyens mettre en œuvre ?) maisqu'elles sont aussi d'ordre ontologique (quelle est la nature des problèmes pédagogiques à explorer ?), épistémologi que (quelles relations instaurer entre l'objet et le sujet de la recherche ou de l'évaluation ?) et surtout, in fine, de nature éthique (au nom de quoi décider ?).

mailto:jean.jouquan@chu-brest.fr 